Supporting Information for

\title{
Descriptors for Hydrogen Evolution on Single Atom Catalysts in Nitrogen-doped Graphene
}

\author{
Victor Fung, ${ }^{1,2 \#}$ Guoxiang $\mathrm{Hu},{ }^{1,2 \#}$ Zili $\mathrm{Wu},{ }^{2,3}$ and De-en Jiang ${ }^{1 *}$ \\ ${ }^{1}$ Department of Chemistry, University of California, Riverside, California 92521, US \\ ${ }^{2}$ Center for Nanophase Materials Sciences, Oak Ridge National Laboratory, Oak Ridge, \\ Tennessee 37831 \\ ${ }^{3}$ Chemical Sciences Division, Oak Ridge National Laboratory, Oak Ridge, Tennessee 37831
}

*To whom correspondence should be addressed. E-mail: djiang@ucr.edu. 
Table S1. Properties and descriptors of transition-metal single-atom catalysts in nitrogen-doped graphene (see footnotes for definitions of each descriptor).

\begin{tabular}{|c|c|c|c|c|c|c|c|c|c|c|c|c|}
\hline Size & atom & $\mathrm{G}_{\mathrm{H}}(\mathrm{eV})$ & $\begin{array}{l}\text { rcov } \\
(\AA)\end{array}$ & $\operatorname{rd}(\AA \AA)$ & $\begin{array}{l}\text { Ef } \\
\text { (eV/atom) }\end{array}$ & $\mathrm{IE}$ & EN & $\varepsilon_{d}(e V)$ & $n$ & $q(e)$ & $d_{o c c}$ & $\mathrm{~d}_{\text {unocc }}$ \\
\hline \multirow{27}{*}{$\begin{array}{l}\text { Periodic } \\
\text { Graphene }\end{array}$} & Sc & -0.190 & 1.700 & 0.310 & -8.282 & 6.561 & 1.360 & 1.064 & 3 & 1.640 & 0.85 & 4.17 \\
\hline & $\mathrm{Ti}$ & -0.500 & 1.600 & 0.280 & -8.326 & 6.827 & 1.540 & 0.998 & 4 & 1.404 & 0.83 & 3.58 \\
\hline & V & -0.270 & 1.530 & 0.260 & -7.494 & 6.746 & 1.630 & 1.316 & 5 & 1.341 & 1.22 & 2.57 \\
\hline & $\mathrm{Cr}$ & 0.310 & 1.390 & 0.250 & -6.604 & 6.766 & 1.660 & 0.944 & 6 & 1.263 & 2.36 & 0.98 \\
\hline & $\mathrm{Mn}$ & 0.430 & 1.390 & 0.230 & -6.520 & 7.434 & 1.550 & -0.513 & 7 & 1.279 & 1.33 & 2.46 \\
\hline & $\mathrm{Fe}$ & 0.250 & 1.320 & 0.220 & -7.360 & 7.902 & 1.830 & -0.846 & 8 & 1.080 & 2.98 & 1.23 \\
\hline & Co & 0.150 & 1.260 & 0.210 & -7.562 & 7.880 & 1.880 & -0.573 & 9 & 0.887 & 4.80 & 1.30 \\
\hline & $\mathrm{Ni}$ & 1.590 & 1.240 & 0.195 & -7.699 & 7.639 & 1.910 & -1.750 & 10 & 0.844 & 1.91 & 0.58 \\
\hline & $\mathrm{Cu}$ & 1.680 & 1.320 & 0.185 & -5.097 & 7.726 & 1.900 & -2.748 & 11 & 0.928 & 0.47 & 0.45 \\
\hline & $\mathrm{Zn}$ & 0.760 & 1.220 & 0.175 & -3.174 & 9.394 & 1.650 & -3.581 & 12 & 1.159 & 0.52 & 0.01 \\
\hline & $\mathrm{Zr}$ & -0.900 & 1.750 & 0.540 & -8.071 & 6.634 & 1.330 & 1.749 & 4 & 1.788 & 0.16 & 1.15 \\
\hline & $\mathrm{Nb}$ & -0.760 & 1.640 & 0.510 & -8.042 & 6.758 & 1.600 & 1.132 & 5 & 1.646 & 0.35 & 0.69 \\
\hline & Mo & -0.910 & 1.540 & 0.490 & -5.661 & 7.092 & 2.160 & 0.820 & 6 & 1.310 & 0.99 & 2.06 \\
\hline & Tc & -0.460 & 1.470 & 0.455 & -6.988 & 7.275 & 1.900 & 0.006 & 7 & 1.264 & 2.84 & 1.37 \\
\hline & $\mathrm{Ru}$ & -0.560 & 1.420 & 0.450 & -7.135 & 7.361 & 2.220 & -0.191 & 8 & 0.951 & 3.54 & 1.46 \\
\hline & $\mathrm{Rh}$ & -0.170 & 1.420 & 0.420 & -7.579 & 7.459 & 2.280 & -0.056 & 9 & 0.619 & 4.47 & 0.46 \\
\hline & $\mathrm{Pd}$ & 1.840 & 1.390 & 0.400 & -5.704 & 8.337 & 2.200 & -2.046 & 10 & 0.703 & 1.01 & 0.09 \\
\hline & $\mathrm{Ag}$ & 0.730 & 1.450 & 0.385 & -1.862 & 7.576 & 1.930 & -4.184 & 11 & 0.853 & 0.09 & 0.57 \\
\hline & $\mathrm{Cd}$ & -0.060 & 1.440 & 0.370 & -1.185 & 8.994 & 1.690 & -2.385 & 12 & 0.984 & 0.15 & 0.02 \\
\hline & $\mathrm{Hf}$ & -1.040 & 1.750 & 0.630 & -9.420 & 6.824 & 1.300 & 1.392 & 4 & 1.589 & 0.36 & 2.45 \\
\hline & $\mathrm{Ta}$ & -1.080 & 1.700 & 0.605 & -8.892 & 7.887 & 1.500 & 0.841 & 5 & 1.645 & 0.73 & 2.46 \\
\hline & W & -1.450 & 1.620 & 0.590 & -7.101 & 7.980 & 2.360 & 0.096 & 6 & 1.558 & 1.91 & 2.01 \\
\hline & $\mathrm{Re}$ & -0.910 & 1.510 & 0.565 & -6.599 & 7.877 & 1.900 & -0.055 & 7 & 1.379 & 1.42 & 1.93 \\
\hline & Os & -0.730 & 1.440 & 0.543 & -7.784 & 8.706 & 2.200 & -0.262 & 8 & 1.179 & 3.59 & 0.91 \\
\hline & Ir & -0.300 & 1.410 & 0.526 & -8.361 & 9.121 & 2.200 & -0.135 & 9 & 0.720 & 4.42 & 0.42 \\
\hline & $\mathrm{Pt}$ & 1.490 & 1.360 & 0.510 & -7.677 & 9.017 & 2.280 & -1.487 & 10 & 0.715 & 2.33 & 0.14 \\
\hline & $\mathrm{Au}$ & 2.240 & 1.360 & 0.488 & -2.953 & 9.225 & 2.540 & -3.348 & 11 & 0.918 & 0.02 & 0.57 \\
\hline \multirow[t]{7}{*}{$\begin{array}{l}\text { Large } \\
\text { nanographene }\end{array}$} & $\mathrm{Ti}$ & -0.498 & 1.600 & 0.280 & -8.232 & 6.827 & 1.540 & 0.688 & 4 & 1.345 & 1.12 & 3.17 \\
\hline & $\mathrm{V}$ & -0.270 & 1.530 & 0.260 & -7.494 & 6.746 & 1.630 & 0.890 & 5 & 1.275 & 1.92 & 2.12 \\
\hline & $\mathrm{Cr}$ & 0.351 & 1.390 & 0.250 & -6.809 & 6.766 & 1.660 & 0.826 & 6 & 1.212 & 2.43 & 0.37 \\
\hline & $\mathrm{Mn}$ & 0.436 & 1.390 & 0.230 & -6.725 & 7.434 & 1.550 & -0.103 & 7 & 1.226 & 1.28 & 1.61 \\
\hline & $\mathrm{Fe}$ & 0.285 & 1.320 & 0.220 & -7.658 & 7.902 & 1.830 & -0.267 & 8 & 1.058 & 3.49 & 1.55 \\
\hline & Co & 0.170 & 1.260 & 0.210 & -7.807 & 7.880 & 1.880 & -0.389 & 9 & 0.855 & 5.57 & 0.80 \\
\hline & $\mathrm{Ni}$ & 1.590 & 1.240 & 0.195 & -7.699 & 7.639 & 1.910 & -1.594 & 10 & 0.800 & 4.36 & 1.15 \\
\hline
\end{tabular}




\begin{tabular}{|c|c|c|c|c|c|c|c|c|c|c|c|c|}
\hline & $\mathrm{Cu}$ & 1.867 & 1.320 & 0.185 & -5.243 & 7.726 & 1.900 & -2.538 & 11 & 0.891 & 0.59 & 0.57 \\
\hline & $\mathrm{Zn}$ & 1.090 & 1.220 & 0.175 & -3.572 & 9.394 & 1.650 & -4.913 & 12 & 1.115 & 0.54 & 0.01 \\
\hline & $\mathrm{Zr}$ & -0.900 & 1.750 & 0.540 & -8.071 & 6.634 & 1.330 & 0.669 & 4 & 1.775 & 0.38 & 0.57 \\
\hline & $\mathrm{Nb}$ & -0.739 & 1.640 & 0.510 & -7.893 & 6.758 & 1.600 & 0.781 & 5 & $\begin{array}{l}1.529 \\
\end{array}$ & 0.09 & 0.91 \\
\hline & Mo & -0.782 & 1.540 & 0.490 & -6.190 & 7.092 & 2.160 & 0.093 & 6 & 1.214 & 2.03 & 1.31 \\
\hline & $\begin{array}{l}\text { TC } \\
\end{array}$ & -0.391 & 1.470 & 0.455 & -6.696 & 7.275 & 1.900 & -0.570 & 7 & 1.203 & 3.27 & 1.83 \\
\hline & $\mathrm{Ru}$ & -0.524 & 1.420 & 0.450 & -7.349 & 7.361 & 2.220 & -0.874 & 8 & 0.963 & 4.15 & 1.17 \\
\hline & $\mathrm{Rh}$ & -0.200 & 1.420 & 0.420 & -7.649 & 7.459 & 2.280 & -0.730 & 9 & 0.672 & 4.55 & 0.50 \\
\hline & $\mathrm{Pd}$ & 1.900 & 1.390 & 0.400 & -5.704 & 8.337 & 2.200 & -2.065 & 10 & 0.680 & 2.07 & 0.11 \\
\hline & $\mathrm{Ag}$ & 0.970 & 1.450 & 0.385 & -2.036 & 7.576 & 1.930 & -4.061 & 11 & 0.772 & 0.11 & 0.49 \\
\hline & $\mathrm{Cd}$ & -0.080 & 1.440 & 0.370 & -1.185 & 8.994 & 1.690 & -2.003 & 12 & 1.061 & 0.47 & 0.02 \\
\hline & $\mathrm{Hf}$ & -1.162 & 1.750 & 0.630 & -9.163 & 6.824 & 1.300 & 0.682 & 4 & 1.558 & 0.39 & 2.07 \\
\hline & $\overline{\mathrm{Ta}}$ & -1.085 & 1.700 & 0.605 & $\begin{array}{l}-8.734 \\
\end{array}$ & 7.887 & 1.500 & 0.217 & 5 & 1.579 & 1.13 & 2.43 \\
\hline & W & -1.360 & 1.620 & 0.590 & -7.101 & 7.980 & 2.360 & -0.047 & 6 & 1.485 & 1.04 & 2.35 \\
\hline & $\operatorname{Re}$ & -0.752 & 1.510 & 0.565 & -6.938 & 7.877 & 1.900 & $\begin{array}{l}-0.534 \\
\end{array}$ & 7 & 1.339 & 1.87 & 1.24 \\
\hline & Os & -0.670 & 1.440 & 0.543 & -8.200 & 8.706 & 2.200 & -0.195 & 8 & 0.940 & 3.93 & 1.04 \\
\hline & Ir & -0.300 & 1.410 & 0.526 & -8.698 & 9.121 & 2.200 & -0.899 & 9 & 0.773 & 3.14 & 0.47 \\
\hline & $\mathrm{Pt}$ & 1.490 & 1.360 & 0.510 & -7.677 & 9.017 & 2.280 & -1.850 & 10 & 0.707 & 2.15 & 0.13 \\
\hline & $\mathrm{Au}$ & 2.052 & 1.360 & 0.488 & -2.853 & 9.225 & 2.540 & -3.645 & 11 & 0.902 & 0.01 & 0.90 \\
\hline Medium & $\mathrm{Ti}$ & -0.520 & 1.600 & 0.280 & -8.592 & 6.827 & 1.540 & 0.328 & 4 & 1.388 & 0.77 & 4.12 \\
\hline & V & -0.260 & 1.530 & 0.260 & -7.870 & 6.746 & 1.630 & 0.175 & 5 & 1.316 & 2.12 & 2.01 \\
\hline & $\mathrm{Cr}$ & 0.330 & 1.390 & 0.250 & -6.980 & 6.766 & 1.660 & 0.360 & 6 & 1.221 & 2.97 & 0.37 \\
\hline & $\mathrm{Mn}$ & 0.420 & 1.390 & 0.230 & -6.987 & 7.434 & 1.550 & -0.384 & 7 & 1.256 & 1.58 & 1.42 \\
\hline & $\mathrm{Fe}$ & 0.260 & 1.320 & 0.220 & -7.846 & 7.902 & 1.830 & -0.648 & 8 & 1.083 & 3.52 & 1.77 \\
\hline & Co & 0.160 & 1.260 & 0.210 & -7.948 & 7.880 & 1.880 & $\begin{array}{l}-0.656 \\
\end{array}$ & 9 & 0.896 & 4.35 & 1.54 \\
\hline & $\mathrm{Ni}$ & 1.570 & 1.240 & 0.195 & -8.125 & 7.639 & 1.910 & \begin{tabular}{|c|}
-1.271 \\
\end{tabular} & 10 & 0.808 & 4.62 & 1.28 \\
\hline & $\mathrm{Cu}$ & 1.830 & 1.320 & 0.185 & -5.473 & 7.726 & 1.900 & -2.626 & 11 & 0.893 & 0.52 & 0.58 \\
\hline & $\mathrm{Zn}$ & 1.150 & 1.220 & 0.175 & -3.621 & 9.394 & 1.650 & -4.639 & 12 & 1.102 & 0.55 & 0.01 \\
\hline & $\mathrm{Zr}$ & -0.920 & 1.750 & 0.540 & -8.071 & 6.634 & 1.330 & 0.782 & 4 & 1.815 & 0.91 & 1.11 \\
\hline & $\mathrm{Nb}$ & -0.800 & 1.640 & 0.510 & -8.448 & 6.758 & 1.600 & -0.348 & 5 & 1.634 & 0.48 & 0.71 \\
\hline & Mo & -0.960 & 1.540 & 0.490 & -6.177 & 7.092 & 2.160 & -0.280 & 6 & 1.416 & 1.84 & 1.38 \\
\hline & Tc & -0.390 & 1.470 & 0.455 & -7.595 & 7.275 & 1.900 & -0.745 & 7 & 1.330 & 2.11 & 1.97 \\
\hline & $\mathrm{Ru}$ & -0.460 & 1.420 & 0.450 & -7.851 & 7.361 & 2.220 & \begin{tabular}{l|l}
-0.454 \\
\end{tabular} & 8 & 0.918 & 3.06 & 0.69 \\
\hline & $\mathrm{Rh}$ & -0.230 & 1.420 & 0.420 & -7.976 & 7.459 & 2.280 & -0.436 & 9 & 0.675 & 4.48 & 0.61 \\
\hline & $\mathrm{Pd}$ & 1.880 & 1.390 & 0.400 & -6.270 & 8.337 & 2.200 & -2.407 & 10 & 0.680 & 3.27 & 0.06 \\
\hline & $\mathrm{Ag}$ & 1.120 & 1.450 & 0.385 & -2.438 & 7.576 & 1.930 & -4.026 & 11 & 0.845 & 0.07 & 0.68 \\
\hline & $\mathrm{Cd}$ & -0.090 & 1.440 & 0.370 & -1.185 & 8.994 & 1.690 & -1.685 & 12 & 1.050 & 0.14 & 2.60 \\
\hline & $\mathrm{Hf}$ & -1.180 & 1.750 & 0.630 & -9.796 & 6.824 & 1.300 & 0.737 & 4 & 1.552 & 1.11 & 2.70 \\
\hline & $\mathrm{Ta}$ & -1.100 & 1.700 & 0.605 & -9.378 & 7.887 & 1.500 & 0.171 & 5 & 1.605 & 2.05 & 2.52 \\
\hline & W & -1.350 & 1.620 & 0.590 & -7.877 & 7.980 & 2.360 & -0.092 & 6 & 1.575 & 2.12 & 1.67 \\
\hline & Os & -0.770 & 1.440 & 0.543 & -8.490 & 8.706 & 2.200 & -0.756 & 8 & 0.985 & 3.59 & 0.93 \\
\hline
\end{tabular}




\begin{tabular}{|c|c|c|c|c|c|c|c|c|c|c|c|c|}
\hline & Ir & -0.330 & 1.410 & 0.526 & -8.957 & 9.121 & 2.200 & -1.307 & 9 & 0.836 & 3.58 & 0.60 \\
\hline & $\mathrm{Pt}$ & 1.490 & 1.360 & 0.510 & -8.393 & 9.017 & 2.280 & $\begin{array}{l}-1.265 \\
\end{array}$ & 10 & 0.712 & 1.76 & 0.14 \\
\hline & $\mathrm{Au}$ & 2.250 & 1.360 & 0.488 & -3.789 & 9.225 & 2.540 & -2.734 & 11 & 0.896 & 0.03 & 0.73 \\
\hline \multirow{21}{*}{$\begin{array}{l}\text { Small } \\
\text { nanographene }\end{array}$} & $\mathrm{Ti}$ & -0.424 & 1.600 & 0.280 & -9.150 & 6.827 & 1.540 & 0.792 & 4 & 1.400 & 1.16 & 2.95 \\
\hline & V & -0.030 & 1.530 & 0.260 & -8.780 & 6.746 & 1.630 & 0.436 & 5 & 1.267 & 2.21 & 1.90 \\
\hline & $\mathrm{Cr}$ & 0.427 & 1.390 & 0.250 & -7.821 & 6.766 & 1.660 & 0.783 & 6 & 1.212 & 2.27 & 0.94 \\
\hline & $\mathrm{Mn}$ & 0.473 & 1.390 & 0.230 & -7.683 & 7.434 & 1.550 & -0.402 & 7 & 1.217 & 2.62 & 2.91 \\
\hline & $\mathrm{Fe}$ & 0.315 & 1.320 & 0.220 & -8.671 & 7.902 & 1.830 & -0.270 & 8 & 0.993 & 4.06 & 1.14 \\
\hline & Co & 0.396 & 1.260 & 0.210 & -8.837 & 7.880 & 1.880 & -0.561 & 9 & 0.836 & 5.58 & 0.25 \\
\hline & $\mathrm{Ni}$ & 1.763 & 1.240 & 0.195 & -8.840 & 7.639 & 1.910 & -1.480 & 10 & 0.802 & 4.78 & 1.07 \\
\hline & $\mathrm{Cu}$ & 1.971 & 1.320 & 0.185 & -6.165 & 7.726 & 1.900 & -2.614 & 11 & 0.893 & 0.46 & 0.57 \\
\hline & $\mathrm{Zn}$ & 1.378 & 1.220 & 0.175 & -4.577 & 9.394 & 1.650 & -4.841 & 12 & 1.116 & 0.52 & 0.02 \\
\hline & $\mathrm{Zr}$ & -0.936 & 1.750 & 0.540 & -8.530 & 6.634 & 1.330 & 1.307 & 4 & 1.462 & 0.08 & 1.05 \\
\hline & $\mathrm{Nb}$ & -0.567 & 1.640 & 0.510 & -8.899 & 6.758 & 1.600 & -0.305 & 5 & 1.535 & 0.22 & 1.26 \\
\hline & Mo & -0.526 & 1.540 & 0.490 & -7.286 & 7.092 & 2.160 & 0.138 & 6 & 1.273 & 2.47 & 2.36 \\
\hline & Tc & -0.267 & 1.470 & 0.455 & -8.365 & 7.275 & 1.900 & -0.343 & 7 & 1.206 & 3.22 & 1.72 \\
\hline & $\mathrm{Ru}$ & -0.490 & 1.420 & 0.450 & -8.703 & 7.361 & 2.220 & -0.542 & 8 & 0.910 & 3.36 & 1.19 \\
\hline & $\mathrm{Rh}$ & -0.085 & 1.420 & 0.420 & -8.962 & 7.459 & 2.280 & -0.230 & 9 & 0.651 & 4.67 & 0.37 \\
\hline & $\mathrm{Pd}$ & 1.912 & 1.390 & 0.400 & -6.919 & 8.337 & 2.200 & -1.406 & 10 & 0.679 & 2.76 & 0.07 \\
\hline & $\mathrm{Ag}$ & 2.048 & 1.450 & 0.385 & -3.331 & 7.576 & 1.930 & -4.020 & 11 & 0.773 & 0.03 & 0.45 \\
\hline & $\mathrm{Cd}$ & 0.245 & 1.440 & 0.370 & -2.291 & 8.994 & 1.690 & -2.120 & 12 & 1.069 & 0.43 & 0.01 \\
\hline & $\mathrm{Hf}$ & -1.127 & 1.750 & 0.630 & -9.987 & 6.824 & 1.300 & 0.453 & 4 & 1.571 & 0.54 & 2.16 \\
\hline & $\mathrm{Ta}$ & -1.092 & 1.700 & 0.605 & -9.573 & 7.887 & 1.500 & 0.120 & 5 & 1.605 & 1.01 & 1.94 \\
\hline & W & -0.951 & 1.620 & 0.590 & -8.916 & 7.980 & 2.360 & 0.061 & 6 & 1.483 & 2.00 & 2.25 \\
\hline
\end{tabular}

$G_{H}$ is the free energy of hydrogen adsorption

$r_{\text {cov }}$ is the covalent radius

$r_{d}$ is the Zunger radius of the atomic $d$-orbital

$E_{f}$ is the formation energy of the single atom site

IE is ionization energy

EN is electronegativity

$\varepsilon_{d}$ is the d-band center from -6 to $6 \mathrm{eV}$

$\mathrm{n}$ is number of valence electrons

$q$ is Bader charge

$d_{\text {occ }}$ is number of occupied d states from 0 to $-2 \mathrm{eV}$

$d_{\text {unocc }}$ is number of unoccupied $d$ states from 0 to $2 \mathrm{eV}$ 